\title{
Personalisiertes Lernen als pädagogisch-psychologisches didaktisches Konzept
}

Im vorhergehenden Abschnitt 2.3 wurde bereits angedeutet, dass zurzeit kein einheitliches Verständnis von den Funktionen, Inhalten und Zwecken personalisierten Lernens vorliegt (Chiosso, 2012; OECD, 2006; Prain et al., 2013). Wie in Abschnitt 1.1 einleitend festgehalten wurde, wird personalisiertes Lernen deshalb auch als mehrere Dimensionen beinhaltender "Container-Begriff" (Stebler et al., 2017, S. 5) oder „umbrella term“ (Kallick \& Zmuda, 2017, S. 2) bezeichnet. So nehmen Autorinnen und Autoren, die den Begriff verwenden, in unterschiedlicher Gewichtung Bezug auf etablierte pädagogische Ziele und didaktische Konzepte wie zum Beispiel innere Differenzierung (u. a. Klafki \& Stöcker, 1976), Individualisierung (u. a. Bräu, 2005), individuelle Förderung (Klieme \& Warwas, 2011) oder die bereits oben beschriebenen erweiterten Lehr- und Lernformen respektive Formen des offenen Unterrichts (u. a. Peschel, 2003).

Aufgrund der Vielzahl an Beschreibungen, Charakterisierungen und Definitionen zu personalisiertem Lernen werden nachfolgend im ersten Unterkapitel (Abschnitt 3.1) immer wiederkehrende Bedeutungsaspekte herausgearbeitet. Anschließend werden die der Datenanalyse dieser Arbeit (Studie 2: Teilfragen 2.1 und 2.2; siehe Abschnitt 1.4) zugrunde liegenden fünf Dimensionen personalisierten Lernens (Reusser, 2015; Stebler et al., 2018) beschrieben (Abschnitt 3.2). 


\subsection{Personalisiertes Lernen: Eine begriffliche Annäherung}

Bei einer Literaturrecherche ${ }^{1}$ für die vorliegende Arbeit wurden insgesamt 41 Beschreibungen, Charakterisierungen und Definitionen von personalisiertem Lernen im englischsprachigen und deutschsprachigen Bildungsraum gefunden, woraus sich die folgenden häufig verwendeten Bedeutungsaspekte extrahieren lassen:

- kindorientierter Unterricht,

- fachliche und überfachliche Kompetenzen,

- heterogene Lerngruppen und

- digitale Lernmedien.

Der kindzentrierte Unterricht geht $\mathrm{u}$. a. auf reformpädagogische Unterrichtskonzepte zurück (siehe Abschnitt 2.3). Weil wird davon ausgegangen wird, dass die Schülerinnen und Schüler selbst am besten wissen, wie, wo, mit wem und wann sie lernen können, werden sie als ,active and responsible co-authors of their educational script" (Campbell et al., 2007, S. 138) aufgefasst. Lehrpersonen haben die Aufgabe, eine Lernumgebung zu gestalten, in der die Schülerinnen und Schüler ihre Lernprozesse vermehrt selbst steuern können: „In a personalized learning environment, learning starts with the learner. Learners understand how they learn best so they can become active participants in designing their learning goals along with the teachers" (Bray \& McClaskey, 2015, S. 11). Ziel ist es, die individuellen Begabungen einer jeden Schülerin und eines jeden Schülers zu fördern: „Denn [der personorientierte] Ansatz geht davon aus, dass jedes Kind seine je einmaligen und besonderen Begabungen hat [und] dass alle Kinder und

\footnotetext{
${ }^{1}$ Publikationen: Beach und Dovemark (2009); Bray und McClaskey (2015); Breuninger und Schley (2017); Campbell, Robinson, Neelands, Hewston und Mazzoli (2007); Clarke (2013); Department for Children Schools and Families (2008); Department for Education and Skills (2004a, 2004b); DiMartino und Clarke (2008); EDUCAUSE Learning Initiative (2015); Ferrer (2012); Garrick, Pendergast und Geelan (2017); Hackl (2011); D. Hargreaves (2006a); Järvelä (2006); Kallick und Zmuda (2017); Keamy et al. (2007); Keefe (2007); Kennedy et al. (2014); Kübler (2016); Leadbeater (2005); Miliband (2006); Mincu (2012a); (Mincu, 2012b); Müller (2014, 2015); Murphy (2016); OECD (2013); Patrick, Kennedy und Powell (2013); Prain et al. (2013); Prain et al. (2015a); Rudduck, Brown und Hendy (2006); Schratz und WestfallGreiter (2010); (Stebler et al., 2017, 2018); Stiftung Mercator Schweiz (www.lernkonzepte. ch/hintergrund [letzter Zugriff: 01.01.2021]); Powell und Kusuma-Powell (2011); Teaching and Learning in 2020 Review Group (2006); U.S. Department of Education (2010); UNESCO (2012); Waldrip, Yu und Prain (2016); Watson und Watson (2017); Weigand (2004); Yonezawa et al. (2012); Zmuda et al. (2015); Datum der Recherche: 15. Mai 2017
} 
Heranwachsenden ihren besonderen Begabungen gemäß gefördert und unterstützt werden“ (Weigand, 2004, S. 361). Im englischsprachigen Raum wird in diesem Zusammenhang von einer learner-centered (Keefe, 2007; Watson \& Watson, 2017) oder student-driven (Zmuda et al., 2015) Lernumgebung gesprochen.

In solchen personalisierten Lernumgebungen ist eine Methodenvielfalt charakteristisch. Ein eng geführter Klassenunterricht wird ergänzt mit Lehr- und Lernformen, in denen die Schülerinnen und selbstständig und kooperativ lernen. Sie sollen nicht nur fachliche, sondern auch vermehrt überfachliche Kompetenz erwerben, beispielsweise Ziele setzen, einen Plan erstellen, um diese Ziele zu erreichen, sich selbst motivieren, um diese Ziele zu erreichen, sowie den Arbeitsund Lernprozess reflektieren. Besonders die überfachlichen Kompetenzen werden als besonders bedeutsam erachtet, um die Schülerinnen und Schüler auf eine zukünftige Berufswelt, in der viele Anforderungen und Jobprofile heute noch nicht bekannt sind, vorzubereiten und sie für lebenslanges Lernen zu qualifizieren (Beach \& Dovemark, 2009; Keamy et al., 2007). Gerade sogenannte „Schlüsselkompetenzen“, wie Analysieren, kritisches Denken, Beurteilen, Vergleichen und Evaluieren, sind wichtige Bestandteile vieler Konzepte (Department for Education and Skills, 2004a; Järvelä, 2006).

Personalisierten Lernangeboten wird das Potenzial zugeschrieben, in heterogenen Lerngruppen auf die individuellen Bedürfnisse und Interessen in besonderem $\mathrm{Maß}$ eingehen zu können: „It means taking the care to nurture the unique talents of every pupil“" (Department for Education and Skills, 2004b, S. 5). Dies ist gerade in integrativen respektive inklusiven Lernsettings (u. a. Ferrer, 2012) ein Thema, um allen Schülerinnen und Schülern nachhaltiges Lernen zu ermöglichen:

Who is personalized learning for? It's for students who are culturally diverse, students who are learning English as a second or third language, students with special learning needs, and students with special gifts or talents. In short, personalized learning is for every student, and it servers all students well. (Powell \& Kusuma-Powell, 2011, S. 7)

Des Weiteren werden im Kontext personalisierten Lernens oftmals Bezüge zur Nutzung digitaler Medien hergestellt (Holmes et al., 2018; Petko et al., 2017): „Proponents of personalized learning maintain that IT systems and tools, along with rich data sets and analytics programs, can provide individualized learning pathways to large numbers of students" (EDUCAUSE Learning Initiative, 2015, S. 1). Besonderes Potenzial kommt diesbezüglich Lernprogrammen zu, die Ergebnisse bereits gelöster Lernaufgaben speichern und darauf aufbauend ein individuelles Lernprogramm zusammenstellen, das falsch gelöste Aufgaben wiederholt und bekannte Lerninhalte vertieft (Mincu, 2012a). 


\subsection{Dimensionen personalisierten Lernens}

Wie bereits erwähnt, sind bei der konkreten didaktischen Gestaltung personalisierter Lernumgebungen deutliche Unterschiede erkennbar. Bezüge zu etablierten Konzepten wie beispielsweise innerer Differenzierung (u. a. Klafki \& Stöcker, 1976), Individualisierung (u. a. Bräu, 2005) oder offenem Unterricht (u. a. Peschel, 2003) werden unterschiedlich stark hergestellt. In Anbetracht dessen ist es unmöglich, eine einheitliche Definition auf der Handlungsebene der Lehrpersonen zu formulieren. Einige Autorinnen und Autoren greifen daher auf eher abstraktere Dimensionen zurück. So haben Waldrip et al. (2014) aufbauend auf einem Literaturreview von über 50 politischen und wissenschaftlichen Artikeln zu Praktiken, Charakteristika, Vorgehensweisen sowie Techniken von personalized learning folgende Dimensionen erarbeitet:

self-directed learning (including self-management, desire for learning and selfcontrol), teacher support, personal relevance, shared control, student engagement (emotional, cognitive, behavioural), congruence with planned learning, authenticity, student consultation, transparency, academic efficacy, peer relationships, self-report on disruptive behaviour, individualisation and the opportunity for personal and social development. (Waldrip et al., 2016, S. 172)

Die vorliegende empirische Forschungsarbeit stützt sich auf fünf von Reusser (2015) beschriebenen Dimensionen personalisierten Lernens, die sich an person- und kindorientierten Lehr- und Lernkonzepten sowie an der pädagogischpsychologischen Unterrichtsforschung orientieren (u. a. auch Stebler et al., 2017, 2018). Im Folgenden werden die Dimensionen vorgestellt und mit Zitaten aus der in Abschnitt 3.1 erwähnten Literaturrecherche erläutert.

Erste Dimension: Unterrichtsangebote an Schülerinnen und Schüler respektive Lerngruppen und deren personale Voraussetzungen anpassen.

Personalisierte Lernangebote sind verstärkt auf individuelle Fähigkeiten und Fertigkeiten, Interessen und Motivationslagen der Schülerinnen und Schüler ausgerichtet. Unter Bezugnahme auf verschiedenste Lehr- und Lernformen der inneren Differenzierung, der Individualisierung oder/und der Öffnung des Unterrichts erstellen Lehrpersonen ein Angebot, bei dem die Schülerinnen und Schüler vermehrt selbst wählen, wie, was, womit, wo und mit wem sie lernen möchten (Beach \& Dovemark, 2009; Kübler, 2016; Stebler et al., 2017, 2018). Ein solches Lernsetting versteht sich als ein ,entsprechende[r] Freiraum, [um] den persönlichen Anschluss zu den Lerninhalten und [die] Erschließung der Bedeutung von Lernerfahrungen für das eigene Lernen" (Schratz \& Westfall-Greiter, 2010, S. 26) 
zu ermöglichen. Jede Schülerin und jeder Schüler hat die Möglichkeit, eigene Lernprozesse selbst zu gestalten. Jedoch ist dies abhängig davon, ob und wie die Lehrperson Handlungs- und Autonomiespielräume im Unterricht anbietet und ob die Schülerinnen und Schüler diese auch nutzen können (Stebler et al., 2017).

Zweite Dimension: Personale und soziale Kompetenzen aufbauen und ganzheitliche Förderung der Schülerinnen und Schüler.

Mit personalisiertem Lernen geht ein mehrdimensionales Wirkungsverständnis von Bildung einher (u. a. Lehrplan 21: D-EDK, 2014), bei dem nicht nur der fachliche, sondern auch der überfachliche Kompetenzerwerb relevant ist (Stebler et al., 2017, 2018). Die Schülerinnen und Schüler sollen sich ,self-regulated learning skills and not just knowledge“" (Watson \& Watson, 2017, S. 98) aneignen, die metakognitive, soziale und emotionale Kompetenzen oder Schlüsselkompetenzen wie Analysieren, kritisches Denken, Beurteilen, Vergleichen und Evaluieren (Järvelä, 2006) umfassen: „Enhancing the student's motivation to learn as well as enhancing metacognitive, social, and emotional competencies to foster selfdirection and achieve mastery of knowledge and skills" (Murphy, 2016, S. ii). Ebenso betont wird die Persönlichkeitsförderung, bei der beispielsweise Motive, Einstellungen und Haltungen für lebenslanges Lernen kultiviert werden (Stebler et al., 2017, 2018).

In der einschlägigen Literatur geht personalisiertes Lernen häufig mit alternativen Bewertungsformen zu der rein summativen Leistungsbewertung einher. In diesem Zusammenhang werden Ansprüche auf authentische Bewertung, formative Beurteilung des Lernerfolgs sowie digitale Portfolios genannt (Järvelä, 2006). Ein diesbezüglich bedeutsamer Ansatz ist das assessment for learning (u. a. D. Hargreaves, 2006a; Miliband, 2006): „Assessment that is related to meaningful tasks and includes assessment for and from students" (Keamy et al., 2007, S. 2). Hier werden die Erkenntnisse aus den Leistungsüberprüfungen und anschließenden persönlichen Gesprächen zwischen Lehrperson und Schülerin oder Schüler genutzt, um die Lernprozesse zu beurteilen: „It needs assessment for learning and the use of data and dialogue to diagnose every student's learning needs“ (Miliband, 2006, S. 21).

\section{Dritte Dimension: Selbstgesteuertes Lernen auf eigenen Wegen ermöglichen.}

Den Gedanken, das selbstgesteuerte Lernen auf eigenen Wegen zu ermöglichen, fassen Patrick, Kennedy und Powell (2013, S. 5) wie folgt zusammen: „Personalized learning $[\ldots]$ is students managing their own work calendars and daily schedules to stay on track, so they are free to move through courses at their own pace and have individualized learning paths and intervention plans." Dass 
die Schülerinnen und Schüler ihr eigenes Lernen „managen“ können, begründen einige Autorinnen und Autoren mit dem reformpädagogischen Argument, dass jede und jeder einzelne Lernende am besten wisse, wie sie oder er am besten lerne (u. a. Bray \& McClaskey, 2015; Kallick \& Zmuda, 2017; Prain et al., 2015a). Ein konstruktivistisches Lernverständnis ist hier anschlussfähig: Da Lernprozesse als individuelle Wissenskonstruktionen verstanden werden können und jedes Kind auf eigene Art und Weise interaktiv Lerninhalte versteht und erwirbt, braucht es auch eine hierfür förderliche Lernumgebung: „Learning is not just the successful transfer of knowledge and skills. Learning comes through interaction in which the learner discovers for themselves, reflects on what they have learned and how" (Leadbeater, 2005, S. 7).

Um Lernen auf eigenen Wegen zu ermöglichen, braucht es einen Unterricht mit Autonomie- und Handlungsspielräumen hinsichtlich Themen, Lernwegen, Lernzeit-Taktung und Lernorten. Diese sollen die Schülerinnen und Schüler eigenständig nutzen und auf diese Weise bestenfalls Selbstwirksamkeit erleben (Stebler et al., 2017). Die Intensität und der Umfang des selbstständigen Lernens hängen davon ab, wie gut die Schülerinnen und Schüler mit Lernwerkzeugen vertraut sind und diese zielgerichtet und verantwortungsvoll einsetzen können, und auch davon, wie gut sie selbst ihre eigenen Fähigkeiten und Fertigkeiten realistisch einschätzen können (DiMartino \& Clarke, 2008; Stebler et al., 2017): Sind sie beispielsweise in der Lage, aus verschiedenen Lernaufgaben mit unterschiedlichen Schwierigkeitsgraden die für sie passende auszuwählen?

Vierte Dimension: Kompetenzorientiertes Lernen zur persönlichen Sache machen. Personalisiertes Lernen zielt darauf ab, dass die Schülerinnen und Schüler sich selbst zur Anstrengung und Übernahme von (Mit-)Verantwortung für zielerreichendes Lernen verpflichten und somit eigenverantwortlich lernen (Stebler et al., 2017). Eigenverantwortung im Lernprozess wird auch bei anderen Autorinnen und Autoren aufgeführt: So heißt es bei Campbell et al. (2007, S. 138): „Children constructed as active and responsible co-authors of their educational script“, bei Beach und Dovemark (2009, S. 690) ,private/individual responsibility“ oder bei Breuninger und Schley (2017, S. 35), Herausforderungen im Lernen „eigenverantwortlich zu bewältigen“.

Trotz der Autonomie- und Handlungsspielräume und deren selbstständiger und eigenverantwortlicher Nutzung kommt es beim personalisierten Lernen auch darauf an, Verbindlichkeiten zu schaffen (Müller, 2014). Diese Verbindlichkeiten beziehen sich auf das Setzen von Zielen, welche die Schülerinnen und Schüler selbst oder in Absprache mit der Lehrperson festlegen und welche von den Lehrpersonen überblickt, kontrolliert und eingefordert werden. Diese Dimension setzt 
somit die Bereitschaft voraus, sich an verbindlichen Kompetenzerwartungen und Gütemaßstäben messen zu lassen (Stebler et al., 2017).

Fünfte Dimension: Als Lehrperson und Lerngemeinschaft bildend und unterstützend wirken.

Neben individuellem und eigenständigem Lernen ist die Situierung der Lernenden in einer Lerngemeinschaft eine weitere zentrale Dimension. Bedeutsam für den Lernertrag ist ein ko-produktives und ko-konstruktives Lernen an gemeinsamen Lerngegenständen, bei dem in kooperativer Auseinandersetzung mit fachlichen Problemen Denk- und Verstehensprozesse angeregt werden (Bray \& McClaskey, 2015; Stebler et al., 2017). Hierbei förderlich ist eine wertschätzende Lernbeziehung, in der Lehrpersonen ,ansteckend“ (contagious) wirken: Sie begeistern, muten zu (Stebler et al., 2017) und fördern und fordern die Schülerinnen und Schüler mit kognitiv anspruchsvollen Lernaufgaben, bei denen eigenständig oder/und kooperativ mit anderen Lernenden an herausfordernden Problemstellungen gearbeitet wird (u. a. Reusser, 2005). In der Lernunterstützung geben Lehrpersonen nicht nur direkte Instruktionen, sondern fungieren auch als Mentorinnen und Mentoren sowie als Coachs: „Through these new relationships, teachers can forge new roles with students - as mentors, counselors, instructional coaches - and more easily and accurately individualize their pedagogy, curricula, and assessments of students' instructional needs" (Yonezawa et al., 2012, S. 2). In diesem Zusammenhang wird in der einschlägigen Literatur zu personalized learning oft das Konzept der kognitiven Meisterlehre aufgeführt: modeling, coaching, scaffolding, fading und reflection (Collins, Brown \& Newman, 1989; Reusser \& Pauli, 2015): Die Lehrperson veranschaulicht durch lautes Denken die kognitiven Zieltätigkeiten (z. B. Lösen das eines Problems) (modeling), leitet die Schülerinnen und Schüler bei ihren Problemlöseversuchen an und unterstützt situationsbezogen (coaching). Als „Lerngerüst“ gibt die Lehrperson Hilfestellungen, die schrittweise „zurückgebaut“ werden. Die Schülerinnen und Schüler wiederum lösen sich von der angeleiteten Lerntätigkeit, übernehmen immer mehr Verantwortung und treffen zunehmend eigene Entscheidungen (scaffolding), während die Lehrperson die Lernsteuerung graduell freigibt (fading). Die Schülerinnen und Schüler werden zudem dazu angehalten, ihre Lernprozesse und Lernprodukte zu reflektieren (reflection).

In Konzepten personalisierten Lernens stehen Lehrpersonen einerseits vor der Herausforderung, Strukturen und Möglichkeiten zu schaffen, um die Schülerinnen und Schüler individuell in ihrem Lernen zu begleiten: ,teachers are to take a structured and responsive approach to learners who, with their help and guidance, are 
to look for their own knowledge as learning-partners and develop a lust for lifelong learning“" (Beach \& Dovemark, 2009, S. 690). Anderseits sind Lehrpersonen gefordert, die gesamte Lerngruppen zu führen und beispielsweise ein produktive Lernklima zu schaffen. Dabei ist nicht nur die soziale Interaktion zwischen Lehrpersonen und den Schülerinnen und Schülern, sondern auch diejenige zwischen den Lernenden in kooperativen Lernsettings relevant: „The learner builds a network of peers, experts, and teachers to guide and support their learning“ (Bray \& McClaskey, 2015, S. 9). In Lerngruppen werden Aufgaben kooperativ bearbeitet, Lerninhalte erklärt, Fragen diskutiert, Probleme gelöst etc. Hierfür benötigen die Schülerinnen und Schüler Kompetenzen, die sie dazu befähigen, beispielsweise in Gesprächen mit Mitschülerinnen und Mitschülern auf fremde Gesprächsbeiträge einzugehen und darauf aufbauend eigene Beiträge zu formulieren, die einen Mehrwert für die Lösung der Aufgabe beinhalten (Ferrer, 2012; Waldrip et al., 2016).

Abschließend stellt sich vor diesem Hintergrund die Frage, welche Akteursgruppen unter einer „Lerngemeinschaft“ gefasst werden können. Im Kontext einer pädagogisch-psychologisch begründeten Didaktik in der Tradition von Aebli (1983/2006) und Reusser (2005) ist das Zusammenwirken von Lehrpersonen und Schülerinnen und Schülern zentral. Im Zusammenhang mit der Schul- und Unterrichtsentwicklung ist zudem auch die Kooperation zwischen Lehrpersonen und deren Potenzial zur Professionalisierung von Bedeutung (siehe Abschnitt 4.5.3). Darüber hinaus werden im angloamerikanischen Bildungsraum zu einer (learning) community auch Eltern, Schulleitende sowie lokale Schulbehörden gezählt (Keamy et al., 2007). Im Kontext der in der vorliegenden Arbeit angelegten Schulentwicklungsperspektive werden die letztgenannten Akteursgruppen ebenfalls als Mitglieder einer Lerngemeinschaft gefasst. 
Open Access Dieses Kapitel wird unter der Creative Commons Namensnennung 4.0 International Lizenz (http://creativecommons.org/licenses/by/4.0/deed.de) veröffentlicht, welche die Nutzung, Vervielfältigung, Bearbeitung, Verbreitung und Wiedergabe in jeglichem Medium und Format erlaubt, sofern Sie den/die ursprünglichen Autor(en) und die Quelle ordnungsgemäß nennen, einen Link zur Creative Commons Lizenz beifügen und angeben, ob Änderungen vorgenommen wurden.

Die in diesem Kapitel enthaltenen Bilder und sonstiges Drittmaterial unterliegen ebenfalls der genannten Creative Commons Lizenz, sofern sich aus der Abbildungslegende nichts anderes ergibt. Sofern das betreffende Material nicht unter der genannten Creative Commons Lizenz steht und die betreffende Handlung nicht nach gesetzlichen Vorschriften erlaubt ist, ist für die oben aufgeführten Weiterverwendungen des Materials die Einwilligung des jeweiligen Rechteinhabers einzuholen. 